\title{
Generalized recurrence, compactifications, and the Lyapunov topology
}

\author{
by \\ Ethan Akin (New York) and Joseph Auslander (College Park, MD)
}

\begin{abstract}
We study generalized recurrence for closed relations on locally compact spaces. This includes continuous maps and real flows. The main tools are Lyapunov functions and their compactifications. Under certain conditions it is shown that the Lyapunov functions determine the topology of the space.
\end{abstract}

In [2] a theory of generalized recurrence in dynamical systems is developed by means of continuous real-valued functions on the phase space. Let $\left\{\varphi^{t}\right\}$ be a real action on the locally compact metric space $X$. Let $L$ be a real-valued function on $X$ which is non-decreasing on every orbit: $L\left(\varphi^{t}(x)\right) \geq L(x)$ for $x \in X$ and $t>0$. The generalized recurrent set $\mathcal{R}$ is defined to be those $x \in X$ for which all such "Lyapunov" functions $L$ are constant on the orbit of $x$. Clearly $\mathcal{R}$ contains the non-wandering points (and therefore the ordinary recurrent points). An intrinsic characterization of $\mathcal{R}$ can be obtained by means of prolongational limit sets.

An interesting class of flows are those for which the generalized recurrent set is empty. Such flows are characterized by the existence of a Lyapunov function which is strictly increasing on every orbit: $L\left(\varphi^{t}(x)\right)>L(x)$ for all $x \in X$ and $t>0$. It turns out that in this case the Lyapunov functions separate points, and so determine a Hausdorff topology on $X$. A natural question is whether this "Lyapunov" topology coincides with the original topology of $X$. Equivalently, if $\left\{x_{n}\right\}$ is a sequence in $X, x \in X$ and $L\left(x_{n}\right) \rightarrow$ $L(x)$ for all Lyapunov functions $L$, is it the case that $x_{n} \rightarrow x$ ?

The purpose of this note is to answer this question in the affirmative (and in fact we will obtain a somewhat more general result). This will require an extensive detour into closed relations on locally compact metric spaces, as well as compactifications of such. In fact, we will obtain the corresponding result for closed relations (and as a consequence for continuous maps).

2010 Mathematics Subject Classification: Primary 54H20.

Key words and phrases: closed relation, generalized recurrence, Lyapunov function, real flow. 
1. Relations and generalized recurrence. Recall that a relation on a set $X$ is just a subset of $X \times X$. In particular a self-map of $X$ is a relation. We write $(X, R)$ if $R$ is a relation on $X$. If $C \subset X, R_{C}$ denotes the induced relation on $C, R_{C}=R \cap(C \times C)$.

The inverse relation $R^{-1}$ is defined by $(x, y) \in R^{-1}$ if $(y, x) \in R$.

If $R$ and $S$ are relations on $X, R \circ S$ is the relation defined by $(x, y) \in R \circ S$ if there is $z \in X$ with $(x, z) \in S$ and $(z, y) \in R$. If $R$ and $S$ are maps, this coincides with the usual definition of composition. We write $R^{2}$ for $R \circ R$. For a positive integer $n, R^{n}$ is defined inductively, and $R^{-n}=\left(R^{-1}\right)^{n}$.

Let $|R|=\{x \in X \mid(x, x) \in R\}$. If $R$ is transitive, then $\left(R \cap R^{-1}\right)_{|R|}$ is an equivalence relation on $|R|$ and $1_{X} \cup\left(R \cap R^{-1}\right)$ is an equivalence relation on $X$ (where $1_{X}$ denotes the diagonal, equivalently the identity map on $X$ ).

If $X$ is a topological space, then $R$ is a closed relation if it is a closed subset of $X \times X$. Thus a continuous self-map of $X$ is a closed relation.

In this paper, we will assume that $X$ is a separable locally compact metric space.

The relation $R$ is said to be + proper if $R(A)$ is compact whenever $A$ is compact, and is said to be proper if $R$ and $R^{-1}$ are + proper. Thus a continuous map is always + proper, and our definition of proper coincides with the usual definition for maps (the inverse image of each compact set is compact)..

The composition of closed relations need not be closed. If the space $X$ is locally compact, then sufficient conditions for $R \circ S$ to be closed are that both $R$ and $S$ are closed, and either $R^{-1}$ or $S$ is + proper.

We write $\mathcal{G} R$ for the smallest closed transitive relation containing $R$ (equivalently the intersection of all closed transitive relations containing $R$ ). Note that for a map $f, \mathcal{G} f$ is in general not a map.

The relation $\mathcal{G} R$ can be obtained by an inductive procedure. The orbit of $R$ is defined as $\mathcal{O} R=\bigcup_{i \geq 1} R^{i}$. Let $\mathcal{N} R=\overline{\mathcal{O} R}$. Thus $y \in \mathcal{N} R(x)$ if there are sequences $\left\{x_{n}\right\}$ and $\left\{y_{n}\right\}$ in $X$ with $x_{n} \rightarrow x, y_{n} \rightarrow y$ and $y_{n} \in R^{k_{n}}\left(x_{n}\right)$ for some $k_{n}>0$.

Now let $\mathcal{N}_{0} R=R$ and define inductively, for $\alpha$ an ordinal number, $\mathcal{N}_{\alpha+1} R=\mathcal{N}\left(\mathcal{N}_{\alpha} R\right)$ (so $\left.\mathcal{N}_{1}=\mathcal{N}\right)$. If $\beta$ is a limit ordinal, define $\mathcal{N}_{\beta} R=$ $\overline{\bigcup_{\alpha<\beta} \mathcal{N}_{\alpha} R}$.

The procedure stabilizes at some ordinal, giving the closed transitive relation $\mathcal{G} R$. Since $X$ is a separable metric space this occurs at a countable ordinal.

We define the generalized recurrent set of $(X, R)$ to be $|\mathcal{G} R|$.

Note that if $x$ and $y$ satisfy $(x, y) \in \mathcal{G} R \cap \mathcal{G} R^{-1}$ then both $x$ and $y$ are in $|\mathcal{G} R|$. In this case, we will frequently say that $x$ and $y$ are $\mathcal{G} R \cap \mathcal{G} R^{-1}$ equivalent, even though this relation is not in general an equivalence relation 
(since it may not be reflexive). As is noted above, it is in fact an equivalence relation when restricted to $|\mathcal{G} R|$.

The following remark is elementary, but will be extremely useful.

Lemma 1. Let $R$ be a closed + proper relation. Then $\mathcal{G} R=R \cup \mathcal{G} R \circ R$.

Proof. Clearly the right side is closed, contains $R$, and is contained in $\mathcal{G} R$. Consideration of four cases $(R \circ R, R \circ \mathcal{G} R, \mathcal{G} R \circ R$, and $\mathcal{G} R \circ \mathcal{G} R)$ shows that the right side is transitive. By definition, it coincides with $\mathcal{G} R$.

Lyapunov functions. A Lyapunov function for $(X, R)$ is a continuous real-valued function $L$ on $X$ such that $L(x) \leq L(y)$ for $(x, y) \in R$. (Thus for a map $f$ we have $L(x) \leq L(f(x))$.) Equivalently, if we define the relation $\leq_{L}$ by $\leq_{L}=\{(x, y) \mid L(x) \leq L(y)\}$ then $R \subset \leq_{L}$. Since $\leq_{L}$ is a closed transitive relation, this in turn is equivalent to $\mathcal{G} R \subset \leq_{L}$, and so $L$ is a Lyapunov function for $(X, \mathcal{G} R)$. Clearly a Lyapunov function is constant on $1_{X} \cup\left(\mathcal{G} R \cap \mathcal{G} R^{-1}\right)$-equivalence classes. It will be convenient (and is no loss of generality) to assume that a Lyapunov function has range contained in $[0,1]$.

A collection $\mathcal{L}$ of Lyapunov functions for $(X, R)$ is said to be sufficient if whenever $L(x) \leq L(y)$ for all $L \in \mathcal{L}$ then $(x, y) \in 1_{X} \cup \mathcal{G} R$. Equivalently, if $(x, y) \notin \mathcal{G} R$ with $x \neq y$ then there is an $L \in \mathcal{L}$ with $L(x)>L(y)$. Still another formulation: $1_{X} \cup \mathcal{G} R$ is the intersection of the relations $\leq_{L}$ for $L \in \mathcal{L}$.

Thus $L(x)=L(y)$ for all $L$ in a sufficient family $\mathcal{L}$ if and only if $x$ and $y$ are in the same $1_{X} \cup\left(\mathcal{G} R \cap \mathcal{G} R^{-1}\right)$-equivalence class.

Sufficient families always exist. (In particular, the collection of all Lyapunov functions for $(X, R)$ is sufficient.) This is a consequence of the following theorem, an "Urysohn Lemma" for Lyapunov functions.

Theorem 2. Let $R$ be a closed transitive relation on $X$, and let $A$ and $B$ be disjoint closed subsets of $X$ with $R(A) \subset A$ and $R^{-1}(B) \subset B$. Then there is a Lyapunov function $L: X \rightarrow[0,1]$ which is 1 on $A$ and 0 on $B$.

Theorem 2 will be proved at the end of the paper.

COROllary 3. $(X, R)$ admits a countable sufficient family.

Proof. Suppose that $(x, y) \notin \mathcal{G} R$ and $x \neq y$. Let $S=1_{X} \cup \mathcal{G} R$, let $A=S(x)$, and $B=S^{-1}(y)$. As $S$ is transitive, and $(x, y) \notin S$, the theorem implies the existence of a Lyapunov function with $L(x)=1$ and $L(y)=0$. Since $X$ is separable metric, $X \times X \backslash\left(1_{X} \cup \mathcal{G} R\right)$ satifies the Lindelöf property and we can choose a countable family $\mathcal{L}$ such that $\left\{>_{L} \mid L \in \mathcal{L}\right\}$ covers it.

In the case of a continuous map, an alternative description of the generalized recurrent set can be formulated using Lyapunov functions. 
Let $f: X \rightarrow X$ be continuous, and let $\mathcal{R}=\{x \in X \mid L(f(x))=L(x)\}$ for all Lyapunov functions $L\}$. Clearly $\mathcal{R}$ is closed, and since $L \circ f$ is also a Lyapunov function, $\mathcal{R}$ is $f$-invariant. We will show that $\mathcal{R}$ coincides with the generalized recurrent set for $f$. For this we need the following lemma.

Lemma 4. Let $f: X \rightarrow X$ be continuous, let $y \in \mathcal{G} f(x)$, and let $L$ be a Lyapunov function for $f$. Then $L(y) \geq L(f(x))$.

Proof. Recall that $\mathcal{G} f=f \cup \mathcal{G} f \circ f$. If $y=f(x)$ then of course $L(y)=$ $L(f(x))$. Otherwise $y \in \mathcal{G} f(f(x))$, so $L(y) \geq L(f(x))$.

Theorem 5. Let $f: X \rightarrow X$ be continuous. Then

(i) $|\mathcal{G} f|=\mathcal{R}$ and if $x$ is in $\mathcal{R}$ then $x$ and $f(x)$ are $\mathcal{G} f \cap \mathcal{G} f^{-1}$-equivalent. (Therefore each $\mathcal{G} f \cap \mathcal{G} f^{-1}$-equivalence class is $f$-invariant.)

(ii) If the Lyapunov functions for $(X, f)$ separate points, then $|\mathcal{G} f|$ is empty or consists of fixed points.

Proof. (i) Suppose $x \in|\mathcal{G} f|, x \in \mathcal{G} f(x)$, and let $L$ be a Lyapunov function. By Lemma $4, L(x) \geq L(f(x))$. Since always $L(f(x)) \geq L(x)$, we have $L(f(x))=L(x)$ so $x \in \mathcal{R}$.

For the opposite inclusion, suppose $x \in \mathcal{R}$. Then $L(f(x))=L(x)$ for every Lyapunov function $L$, so $(x, f(x))$ and $(f(x), x)$ are in $\mathcal{G} f$. Thus we have $f(x)=x$ or $(x, x) \in \mathcal{G} f \circ \mathcal{G} f \subset \mathcal{G} f$. In either case $x \in|\mathcal{G} f|$, and $f(x)$ is $\mathcal{G} f \cap \mathcal{G} f^{-1}$-equivalent to $x$.

(ii) If $x \in|\mathcal{G} f|$ then by (i), $L(f(x))=L(x)$ for all Lyapunov functions $L$ so $f(x)=x$.

In general, if $R$ is a closed relation, it is not the case that $|\mathcal{G} R|$ is $R$ invariant. (That is, if $x \in|\mathcal{G} R|$ and $(x, y) \in R$ then $y$ need not be in $|\mathcal{G} R|$.)

Now suppose $\mathcal{L}$ is a sufficient family for $(X, R)$ and $x \neq y$ with $L(x)=$ $L(y)$ for all $L \in \mathcal{L}$. Then $y \in \mathcal{G} R(x)$ and $x \in \mathcal{G} R(y)$. Thus $x$ and $y$ are in $|\mathcal{G} R|$ and lie in the same $\mathcal{G} R \cap \mathcal{G} R^{-1}$-equivalence class.

It follows that a sufficient family of Lyapunov functions separates points if and only if $\mathcal{G} R \cap \mathcal{G} R^{-1} \subset 1_{X}$. (In particular, this latter condition holds if there are no generalized recurrent points.)

Thus in this case such a family (in particular, the collection of all Lyapunov functions) defines a Hausdorff topology on $X$. A natural question is whether this topology coincides with the given topology of $X$. We will show that under certain reasonable conditions this is in fact the case for the collection of all Lyapunov functions, although not necessarily for any sufficient family.

Compactifications. This is accomplished by using a compactification of $(X, R)$. A compactification of $X$ is a continuous map $j: X \rightarrow \hat{X}$ where $\hat{X}$ 
is a compact metric space and $j(X)$ is dense in $\hat{X}$. If $R$ is a relation on $X$, then $\hat{R}$ is the closure of $j(R)$ in $\hat{X} \times \hat{X}$.

For a general theory of compactifications, see Akin and Auslander [2].

The compactification is good if $j$ is a homeomorphism onto its image. In this case we identify $X$ with $j(X)$, and (since $X$ is locally compact) we may regard $X$ as an open dense subspace of $\hat{X}$, and $\hat{R}$ is the closure of $R$.

If $\mathcal{L}$ is a countable sufficient family of Lyapunov functions we construct a compactification of $(X, R)$ by the following standard method. Let $\mathcal{S}$ be $\mathcal{L}$ together with a countable collection of continuous functions into $[0,1]$ of compact support such that the interiors of the supports form a basis for $X$. Define $\theta: X \rightarrow[0,1]^{\mathcal{S}}$ by $(\theta(x))_{s}=s(x)$ for $s \in \mathcal{S}$, and let $\hat{X}$ be the closure of $\theta(X)$ in $[0,1]^{\mathcal{S}}$. Clearly $X$ is compact and it is metrizable because $\mathcal{S}$ is countable. Since the supports of the functions in $\mathcal{S}$ form a basis the compactification is good. The topology is independent of the choice of functions of compact support, and we refer to $(\hat{X}, \hat{R})$ as the $\mathcal{L}$-compactification of $(X, R)$.

If $f$ is a continuous self-map of $X$, then $L \circ f$ is a Lyapunov function whenever $L$ is, and if in addition $f$ is proper, then $u \circ f$ has compact support whenever $u$ does. Hence, if $f$ is a proper continuous map then we can choose $\mathcal{S}$ so that $u \in \mathcal{S}$ implies $u \circ f \in \mathcal{S}$ (and conversely if $f$ is a homeomorphism). In that case $\hat{f}$ is a continuous map (respectively a homeomorphism) on $\hat{X}$.

Clearly, a Lyapunov function for $(\hat{X}, \hat{R})$ restricts to one for $(X, R)$. Moreover, if $L \in \mathcal{L}$, then $L$ extends to a Lyapunov function for $(\hat{X}, \hat{R})$ by projection on the $L$ th coordinate. Thus we may regard the members of $\mathcal{L}$ as Lyapunov functions for $(\hat{X}, \hat{R})$. (In general, there are Lyapunov functions for $(\hat{X}, \hat{R})$ in addition to those in $\mathcal{L}$.)

Lemma 6. Let $\mathcal{L}$ be a countable sufficient family of Lyapunov functions for $(X, R)$ and let $(\hat{X}, \hat{R})$ be the $\mathcal{L}$-compactification of $(X, R)$. Then

(i) If $\hat{x}$ and $\hat{y}$ are distinct points of $\hat{X} \backslash X$, then $L(\hat{x}) \neq L(\hat{y})$ for some $L \in \mathcal{L}$. (Therefore $\hat{x}$ and $\hat{y}$ are in different $\mathcal{G} \hat{R} \cap \mathcal{G} \hat{R}^{-1}$-equivalence classes.)

(ii) $\left(1_{X} \cup \mathcal{G} \hat{R}\right) \cap(X \times X)=1_{X} \cup \mathcal{G R}$.

Proof. (i) $\mathcal{L}$ together with the continuous functions with compact suppport generate the continuous functions on $\hat{X}$. Hence if $\hat{x}$ and $\hat{y}$ are in $\hat{X} \backslash X$ with $\hat{x} \neq \hat{y}$ they are distinguished by some such function. But if $s$ has compact support, $s(\hat{x})$ and $s(\hat{y})$ are both 0 . Therefore $L(\hat{x}) \neq L(\hat{y})$ for some $L \in \mathcal{L}$.

(ii) Let $(x, y) \in\left(1_{X} \cup \mathcal{G} \hat{R}\right) \cap(X \times X)$, and let $L \in \mathcal{L}$. Since $L$ may be regarded as a Lyapunov function for $(\hat{X}, \hat{R})$, we have $L(x) \leq L(y)$. Since this holds for every $L$ in the sufficient family $\mathcal{L}$, it follows that $(x, y) \in 1_{X} \cup \mathcal{G} R$. 
A compactification $(\hat{X}, \hat{R})$ satisfying the condition in (ii) of Lemma 6 , $\left(1_{X} \cup \mathcal{G} \hat{R}\right) \cap(X \times X)=1_{X} \cup \mathcal{G} R$, is said to be almost dynamic. This occurs precisely when the Lyapunov functions for $(\hat{X}, \hat{R})$ restrict to a sufficient family for $(X, R)$.

The compactification will be called dynamic if it satisfies the stronger condition $\mathcal{G} \hat{R} \cap(X \times X)=\mathcal{G} R$. The only difference between the two conditions is that in the almost dynamic but not dynamic case we may have, for some $x \in X, x \notin|\mathcal{G} R|$ but $x \in|\mathcal{G} \hat{R}|$.

The following theorem describes the $\mathcal{G} \hat{R} \cap \mathcal{G} \hat{R}^{-1}$-equivalence classes.

THEOREM 7. Let $\mathcal{L}$ be a countable sufficient family of Lyapunov functions for $(X, R)$ and let $(\hat{X}, \hat{R})$ be the $\mathcal{L}$-compactification of $(X, R)$. Let $\hat{E}$ be a $\mathcal{G} \hat{R} \cap \mathcal{G} \hat{R}^{-1}$-equivalence class and let $E=\hat{E} \cap X$. Then exactly one of the following occurs:

(i) $\hat{E}$ consists of a single point of $\hat{X} \backslash X($ so $E=\emptyset)$.

(ii) $E \subset|\mathcal{G} R|$ and is a non-compact $\mathcal{G} R \cap \mathcal{G} R^{-1}$-equivalence class with $\hat{E}$ its one-point compactification.

(iii) $\hat{E}=E \subset|\mathcal{G} R|$ and is a compact $\mathcal{G} R \cap \mathcal{G} R^{-1}$-equivalence class.

(iv) $E$ is non-empty and compact and $\hat{E} \backslash E$ is a single point $\{\hat{x}\}$, so $\hat{E}$ is the disjoint union of $E$ and $\{\hat{x}\}$.

The proof is almost immediate. The only problem occurs in case (iii) and in fact the conclusion is clear when $\hat{E}=E$ contains more than one point. If $\hat{E}=\{x\}$ a singleton with $x \in X$, it is necessary to show that $x \in|\mathcal{G} R|$.

This is a consequence of the following lemma:

Lemma 8. Suppose $x, y \in X$ with $(x, y) \in \mathcal{G} \hat{R}$. Then either $(x, y) \in \mathcal{G} R$ or there is a $\hat{z} \in \hat{X} \backslash X$ such that $(x, \hat{z})$ and $(\hat{z}, y)$ are in $\mathcal{G} \hat{R}$.

In particular, if $x \in X$ with $x \in|\mathcal{G} \hat{R}|$ then either $x \in|\mathcal{G} R|$ or there is a $\hat{z} \in \hat{X} \backslash X$ such that $x$ and $\hat{z}$ are in the same $\mathcal{G} \hat{R} \cap \mathcal{G} \hat{R}^{-1}$-equivalence class.

Proof. Let $A=\{(x, y) \in \mathcal{G} \hat{R} \mid$ at least one of $x, y$ is in $\hat{X} \backslash X\}, B=$ $\{(x, y) \in X \times X \mid(x, \hat{z}),(\hat{z}, y) \in \mathcal{G} \hat{R}$ for some $\hat{z} \in \hat{X} \backslash X\}$, and let $R^{*}=$ $\mathcal{G} R \cup A \cup B$.

Then $\hat{R} \subset R^{*} \subset \mathcal{G} \hat{R}$, and $R^{*}$ is closed and transitive, so $R^{*}=\mathcal{G} \hat{R}$.

Case (iv) is somewhat surprising, and in fact it is precisely when it occurs that the Lyapunov functions may separate points, but fail to determine the topology of the space.

The Lyapunov topology. As was mentioned above, when the Lyapunov functions separate points, they determine a Hausdorff topology. This can occur when there are no generalized recurrent points, or more generally there are generalized recurrent points, but for such points their $\mathcal{G} R \cap \mathcal{G} R^{-1}$ equivalence classes consist only of the point (so in the case of a continuous 
map, such a point is a fixed point). The question is whether in such cases this "Lyapunov" topology coincides with the original topology. We will show that in the first case it always does, and if $R$ is + proper it does in the second case as well. The two proofs are quite different.

The following lemma is the main step in dealing with the case of no generalized recurrent points.

LEMma 9. Let $R$ be a closed relation on $X$ and suppose $x \in X$ is not a generalized recurrent point. Then

(i) There is a compact neighborhood $U$ of $x$ such that $\mathcal{G} R(U) \cap \mathcal{G} R^{-1}(U)$ $=\emptyset$.

(ii) Let $U$ be as in (i). Let $L_{0}$ be a Lyapunov function with $0 \leq L_{0} \leq 1$ which is 1 on $\mathcal{G} R(U)$ and 0 on $\mathcal{G} R^{-1}(U)$, and let $\mathcal{L}$ be a countable sufficient family which contains $L_{0}$. Let $(\hat{X}, \hat{R})$ be the $\mathcal{L}$-compactification of $(X, R)$. Then there is no $z \in \hat{X}$ which is in the same $\mathcal{G} \hat{R} \cap \mathcal{G} \hat{R}^{-1}$-equivalence class as $x$.

(iii) $x$ is not a generalized recurrent point for $(\hat{X}, \hat{R})$.

Proof. (i) If not, then this intersection would be non-empty for every compact neighborhood $U$ of $x$, so by transitivity $\mathcal{G} R(U) \cap U \neq \emptyset$ for every such $U$. That is, there are points $y, z \in U$ with $(y, z) \in \mathcal{G} R$. Since this holds for all neighborhoods $U$ of $x$, taking limits we obtain $(x, x) \in \mathcal{G} R$. That is, $x \in|\mathcal{G} R|$.

(ii) Note that an $L_{0}$ with the stated property exists by Theorem 2 .

We will show that if there were such a $z$ (so $\left.z \in \mathcal{G} \hat{R}(x) \cap \mathcal{G} \hat{R}^{-1}(x)\right)$ we would have $L_{0}(z)$ equal to both 0 and 1 . By Lemma $1, \mathcal{G} \hat{R}=\hat{R} \cup(\mathcal{G} \hat{R} \circ \hat{R})$. If $z \in \hat{R}(x)$, then there are $\left(x_{n}, z_{n}\right) \in R$ with $\left(x_{n}, z_{n}\right) \rightarrow(x, z)$. We may suppose $x_{n} \in U$. Then $z_{n} \in R(U) \subset \mathcal{G} R(U)$ and $L_{0}\left(z_{n}\right)=1$, so $L_{0}(z)=1$. If $z \in \mathcal{G} \hat{R}(\hat{R}(x))$, then $z \in \mathcal{G} \hat{R}\left(x^{\prime}\right)$ where $x^{\prime} \in \hat{R}(x)$ so as above $L_{0}\left(x^{\prime}\right)=1$. Since $L_{0}$ is a Lyapunov function for $\hat{R}$, we have $L_{0}(z)=1$.

Now let $R^{\prime}=R^{-1}$ and let $L_{0}^{\prime}=1-L_{0}$. Then $L_{0}^{\prime}$ is a Lyapunov function for $R^{\prime}$ and $z \in \mathcal{G} \hat{R}^{\prime}(x)$. Moreover $L_{0}^{\prime}=1$ on $\mathcal{G} R^{\prime}(U)$. The argument in the previous paragraph yields $L_{0}^{\prime}(z)=1$, so $L_{0}(z)=0$.

(iii) follows from (ii) and Lemma 8.

COROLlary 10. Let $\mathcal{L}$ be a sufficient family of Lyapunov functions for $(X, R)$ with the property that if $x \notin|\mathcal{G} R|$ there is an $L \in \mathcal{L}$ as in (ii) of Lemma 9. Then the $\mathcal{L}$-compactification $(\hat{X}, \hat{R})$ is dynamic.

Proof. Suppose $(x, y) \in \mathcal{G} \hat{R} \cap(X \times X)$. If $x \neq y$ then (since the compactification is almost dynamic) we have $(x, y) \in \mathcal{G} R$. Otherwise $x \in|\mathcal{G} \hat{R}|$ so by Lemma $9, x \in|\mathcal{G} R|$.

We note that since $X_{0}=X \backslash|\mathcal{G} R|$ is Lindelöf, we can find a countable collection of compacta contained in $X_{0}$ whose interiors cover $X_{0}$. Hence we 
can find a countable sufficient family as is required in the hypothesis of Corollary 10.

TheOREM 11. Let $R$ be a closed relation on $X$ such that $(X, R)$ has no generalized recurrent points. Then the topology defined by the Lyapunov functions coincides with the original topology of $X$.

Proof. Let $\mathcal{L}$ be the collection of all Lyapunov functions for $(X, R)$, and let $\mathcal{L}_{0}$ be a countable sufficient family which satisfies the conditions of Corollary 10 . Let $(\hat{X}, \hat{R})$ be the (metrizable and dynamic) $\mathcal{L}_{0}$-compactification of $(X, R)$. Let $\hat{\mathcal{L}}$ be the Lyapunov functions for $(\hat{X}, \hat{R})$ and let $\hat{\mathcal{L}}_{0}$ be a countable uniformly dense subfamily of $\hat{\mathcal{L}}$.

Now the topology of $X$ is finer than the topology defined by $\mathcal{L}$, which in turn is finer than the topology defined by (the restrictions of) $\hat{\mathcal{L}}_{0}$. Thus it suffices to show that the latter topology agrees with the original one on $X$. Since $\hat{\mathcal{L}}_{0}$ is countable, this topology is metrizable. If the $\hat{\mathcal{L}}_{0}$ topology did not coincide with the original topology, there would be a sequence $\left\{x_{n}\right\}$ with no convergent subsequence and an $x \in X$ such that $L\left(x_{n}\right) \rightarrow L(x)$ for every $L \in \hat{\mathcal{L}}_{0}$. We may suppose that $\left\{x_{n}\right\}$ converges to $z \in \hat{X} \backslash X$. Since the compactification is dynamic, and $|\mathcal{G} R|=\emptyset$, the points $x$ and $z$ are not $\mathcal{G} \hat{R} \cap \mathcal{G} \hat{R}^{-1}$-equivalent, so there is an $L^{*} \in \hat{\mathcal{L}}$ such that $L^{*}(z) \neq L^{*}(x)$. We may choose $L^{*} \in \hat{\mathcal{L}}_{0}$, since the latter is dense in $\hat{\mathcal{L}}$. It follows that $L^{*}\left(x_{n}\right)$ does not converge to $L^{*}(x)$.

Now we turn to the case where the relation $R$ is + proper, and the Lyapunov functions separate points. The proof that they determine the topology of $X$ depends on several lemmas about closed relations on compact spaces, some of which concern the chain relation.

(If $R$ is not + proper, it is possible that the Lyapunov functions separate points, but do not determine the original topology. An example will be given below.)

We recall the definition of the chain relation for a closed relation $R$ on a compact space. If $\varepsilon \geq 0$ an $\varepsilon$-chain from $x$ to $y$ is a finite sequence $\left\{x=x_{0}, y_{0}, x_{1}, y_{1}, \ldots, y_{n-1}, x_{n}=y\right\} \quad$ with $\left(x_{i}, y_{i}\right) \in R$ and $d\left(y_{i-1}, x_{i}\right) \leq \varepsilon$. (In the case of a map $f, y_{i}=f\left(x_{i}\right)$ and $d\left(f\left(x_{i-1}\right), x_{i}\right) \leq \varepsilon$.) The chain relation $\mathcal{C} R$ is defined by $(x, y) \in \mathcal{C} R$ if for every $\varepsilon>0$ there is an $\varepsilon$-chain from $x$ to $y$. Thus $\mathcal{C} R=\bigcap_{\varepsilon>0} \mathcal{O}\left(\overline{V_{\varepsilon}} \circ R\right)$ (where $V_{\varepsilon}=\{(x, y) \mid d(x, y)<\varepsilon\}$ ).

The relation $\mathcal{C} R$ contains $R$ and is closed and transitive, so $\mathcal{G} R \subset \mathcal{C} R$.

We call a set $C \mathcal{G} R$-unrevisited (or just unrevisited) if $\mathcal{G} R(C) \cap \mathcal{G} R^{-1}(C)$ $\subset C$.

Lemma 12. Suppose that $X$ is compact, and $R$ is a closed relation on $X$. Let $C$ be a closed unrevisited subset of $X$. Suppose $D$ is a closed neighborhood 
of $C$. Then $(\mathcal{G} R)_{C} \subset \mathcal{G}\left(R_{D}\right)$. (That is, if $x, y \in C$ with $y \in \mathcal{G} R(x)$ then $(x, y)$ is in the smallest closed transitive relation containing $R_{D}$.)

Moreover $(\mathcal{G} R)_{C} \subset \mathcal{C}\left(R_{C}\right)$. (That is, if $x, y \in C$ with $y \in \mathcal{G} R(x)$ then for every $\varepsilon>0$ there is an $\varepsilon$-chain from $x$ to $y$ all of whose members are in $C$.)

Proof. We show that for every ordinal number $\alpha,\left(\mathcal{N}_{\alpha} R\right)_{C} \subset \mathcal{G}\left(R_{D}\right)$. The proof is by induction on $\alpha$.

If $\alpha=0$ this just says that $R_{C} \subset \mathcal{G}\left(R_{D}\right)$, which is obvious.

Although it is not logically required, we give the proof for $\alpha=1$. Let $\varepsilon>0$ be such that $D$ contains the $\varepsilon$-neighborhood of $C$. Suppose that $x, y \in$ $C$ with $y \in \mathcal{N}_{1} R(x)$. Then there are $x_{n}$ and $y_{n}$ with $y_{n} \in R^{k_{n}}\left(x_{n}\right), x_{n} \rightarrow x$, $y_{n} \rightarrow y$ so there is a 0-chain for $R$ from $x_{n}$ to $y_{n}$. If, for sufficiently large $n$, all the members of this 0 -chain are in $D$ then certainly $(x, y) \in \mathcal{G}\left(R_{D}\right)$. If not, there are $s_{n}$ and $t_{n}$ and $z_{n} \notin D$ with $z_{n} \in R^{s_{n}}\left(x_{n}\right)$ and $y_{n} \in R^{t_{n}}\left(z_{n}\right)$. Let (a subsequence of) $z_{n} \rightarrow z$. Then $d(z, C) \geq \varepsilon$ so $z \notin C$. We then have $z \in \mathcal{G} R(x)$ and $y \in \mathcal{G} R(z)$, which contradicts the assumption that $C$ is unrevisited.

Therefore we have $\left(\mathcal{N}_{1} R\right)_{C} \subset \mathcal{G}\left(R_{D}\right)$.

By compactness, there is a closed unrevisited set $C_{1}$ with $C \subset \operatorname{int} C_{1}$ and $C_{1} \subset \operatorname{int} D$. Since $C_{1}$ is closed unrevisited, and is in the interior of $D$, we may apply the induction hypothesis to $C_{1}$.

Suppose $\alpha$ is a limit ordinal, and that for all $\beta<\alpha$ we have $\left(\mathcal{N}_{\beta} R\right)_{C_{1}} \subset$ $\mathcal{G}\left(R_{D}\right)$. Let $\left(x_{i}, y_{i}\right) \in\left(\mathcal{N}_{\beta_{i}} R\right)_{C_{1}}$ with $\left(x_{i}, y_{i}\right) \rightarrow(x, y)$ and $\beta_{i}<\alpha$. By the induction hypothesis, $\left(x_{i}, y_{i}\right) \in \mathcal{G}\left(R_{D}\right)$ so $(x, y) \in \mathcal{G}\left(R_{D}\right)$.

Now suppose $\alpha=\beta+1$. Let $S=\mathcal{N}_{\beta}$. The induction hypothesis is $S_{C_{1}} \subset \mathcal{G}\left(R_{D}\right)$. Then there are $\left(x_{i}, y_{i}\right) \in\left(S^{k_{i}}\right)_{C_{1}}$ with $\left(x_{i}, y_{i}\right) \rightarrow(x, y)$. For sufficiently large $i$, all the members of this 0-chain (for $S$ ) from $x_{i}$ to $y_{i}$ are in $C_{1}$. (If not, just as in the case $\alpha=1$ above, taking a limit point of those members outside $C_{1}$ we would obtain a contradiction to the assumption that $C$ is unrevisited.) Then by the induction hypothesis, and the transitivity of $\mathcal{G}\left(R_{D}\right)$, we have $\left(x_{i}, y_{i}\right) \in \mathcal{G}\left(R_{D}\right)$ so $(x, y) \in \mathcal{G}\left(R_{D}\right)$.

This completes the induction. It now follows immediately that $(\mathcal{G R})_{C} \subset$ $\mathcal{C}\left(R_{D}\right)$ for every closed neighborhood $D$ of $C$,

From this we may conclude, intersecting over all such neighborhoods $D$, that in fact $(\mathcal{G R})_{C} \subset \mathcal{C}\left(R_{C}\right)$.

This is a consequence of the fact that the chain relation is an upper semicontinuous map on closed relations. While this is proved in [1, Theorem 7.23, p. 138], we will indicate the main steps.

What we show is that given $\varepsilon>0$ there is a $\delta>0$ such that $S \subset V_{\delta} \circ R \circ V_{\delta}$ implies $\mathcal{C} S \subset V_{\varepsilon} \circ \mathcal{C} R \circ V_{\varepsilon}$. This in turn follows from the identity $\mathcal{C} R=$ $\bigcap_{\varepsilon>0} \mathcal{N}\left(V_{\varepsilon} \circ R \circ V_{\varepsilon}\right)$, which is proved in [1, Proposition 1.8, p. 12]. (Recall that $\mathcal{N}$ is the closure of the orbit relation.) So, by compactness, given $\varepsilon>0$ 
there is a $\delta>0$ such that $\mathcal{N}\left(V_{2 \delta} \circ R \circ V_{2 \delta}\right) \subset V_{\varepsilon} \circ \mathcal{C} R \circ V_{\varepsilon}$. Hence if $S \subset V_{\delta} \circ R \circ V_{\delta}$ we have $\mathcal{C} S \subset \mathcal{O}\left(V_{\delta} \circ S\right) \subset \mathcal{O}\left(V_{2 \delta} \circ R \circ V_{2 \delta}\right) \subset \mathcal{N}\left(V_{2 \delta} \circ R \circ V_{2 \delta}\right) \subset V_{\varepsilon} \circ \mathcal{C} R \circ V_{\varepsilon}$.

Applying this to the situation at hand, we see that if $(x, y) \in \mathcal{C}\left(R_{D}\right)$ for every closed neighborhood $D$ of $C$, then $(x, y)$ is in every neighborhood of $\mathcal{C}\left(R_{C}\right)$ so in fact $(x, y) \in \mathcal{C}\left(R_{C}\right)$.

TheOrem 13. Suppose $R$ is + proper, and let $(\hat{X}, \hat{R})$ be an almost $d y$ namic compactification of $(X, R)$. Let $C$ be a compact subset of $X$ with $\mathcal{G} R(C) \cap \mathcal{G} R^{-1}(C) \subset C$. Then $\mathcal{G} \hat{R}(C) \cap \mathcal{G} \hat{R}^{-1}(C) \subset C$. In particular, if $x \in X$ satisfies $\mathcal{G} R(x) \cap \mathcal{G} R^{-1}(x) \subset\{x\}$, then $\mathcal{G} \hat{R}(x) \cap \mathcal{G} \hat{R}^{-1}(x) \subset\{x\}$.

Proof. Let $\hat{C}=\mathcal{G} \hat{R}(C) \cap \mathcal{G} \hat{R}^{-1}(C)$. We need to show that $\hat{C}=C$. Note that $\hat{C}$ is a compact subset of $\hat{X}$ and that $\mathcal{G} \hat{R}(\hat{C}) \cap \mathcal{G} \hat{R}^{-1}(\hat{C}) \subset \hat{C}$.

We first show that $\hat{C} \cap X=C$. Let $z \in \hat{C} \cap X$. Let $x, y \in C$ be such that $z \in \mathcal{G} \hat{R}(x)$ and $y \in \mathcal{G} \hat{R}(z)$. Then $(x, z)$ and $(z, y)$ are in $\left(1_{X} \cup \mathcal{G} \hat{R}\right) \cap(X \times X)=$ $1_{X} \cup G R$. Of course if $(x, z)$ or $(z, y)$ is in $1_{X}$ then $z \in C$, and if $(x, z)$ and $(z, y)$ are in $\mathcal{G}(R)$ then $z \in C$ since $\mathcal{G} R(C) \cap \mathcal{G} R^{-1}(C) \subset C$.

Thus it is sufficient to show that $\hat{C} \subset X$, and in turn for this it is sufficient to show that $\mathcal{G} \hat{R}(C) \subset X$.

Let $x \in C$ and let $z \in \mathcal{G} \hat{R}(x)$. It follows from Lemma 12 that for every $\varepsilon>0$ there is an $\varepsilon$-chain for $R$ from $x$ to $z$ all of whose members are in $\hat{C}$.

Since $R$ is + proper, $C \cup R(C)$ is a compact subset of $X$ and so equals $C \cup \hat{R}(C)$. Let $\varepsilon>0$ be such that $N$, the $\varepsilon$-neighborhood of $C \cup R(C)$, is contained in $X$. Let $x=x_{0}, y_{0}, x_{1}, y_{1}, \ldots, x_{n}, y_{n}=z$ be an $\varepsilon$-chain from $x$ to $z$, so $\left(x_{i}, y_{i}\right) \in \hat{R}$ and $d\left(y_{i-1}, x_{i}\right) \leq \varepsilon$, with $x_{i}, y_{i} \in \hat{C}$. We show that $x_{i}$ and $y_{i}$ are in $X$. Now $x_{0}=x \in C$, so suppose $x_{i} \in C$. Then $y_{i} \in R(C) \subset X$, so $y_{i} \in \hat{C} \cap X=C$ and $x_{i+1} \in N \subset X$ and similarly we have $x_{i+1} \in C$. It follows that $z \in C$.

THEOREM 14. Suppose $R$ is + proper, and suppose $\mathcal{G} R(x) \cap \mathcal{G} R^{-1}(x) \subset$ $\{x\}$ for all $x \in X$ (equivalently, the Lyapunov functions separate the points of $X)$. Then the Lyapunov functions for $(X, R)$ determine the topology of $X$.

Proof. We proceed as in the proof of Theorem 11. If the conclusion were false there would be a sequence $\left\{x_{n}\right\}$ in $X$ and an $x \in X$ such that $L\left(x_{n}\right) \rightarrow L(x)$ for all $L$ in a countable sufficient family. But $\left\{x_{n}\right\}$ converges in $\hat{X}$ to a point $z \in \hat{X} \backslash X$. Since $L(z)=L(x)$ for all such $L$ it follows that $x$ and $z$ are $\mathcal{G} \hat{R} \cup \mathcal{G} \hat{R}^{-1}$-equivalent. But this contradicts Theorem 13 .

Here is a simple example where the Lyapunov functions for $(X, R)$ separate points (equivalently $\mathcal{G} R$ is anti-symmetric) but the topology they define does not coincide with the given topology of $X$. 
Let $X$ be the countable subset of the plane

$$
\begin{aligned}
& \{(0,0),(1,-1),(1 / 2,-1 / 2),(1 / 3,-1 / 3), \ldots\} \\
& \quad \cup\{(1,1),(1 / 2,1 / 2),(1 / 3,1 / 3), \ldots\} \cup\{(-1,1),(-1,2),(-1,3), \ldots\}
\end{aligned}
$$

and let the map $f: X \rightarrow X$ be given by $f(1 / n,-1 / n)=(-1, n), f(-1, n)=$ $(1 / n, 1 / n), f(1 / n, 1 / n)=(1 / n+1,1 / n+1), f(0,0)=(0,0)$. Note that $f$ is not continuous, but it is a closed relation. Moreover $f$ is not + proper, since $f((0,0),(1,-1),(1 / 2,-1 / 2), \ldots)$ is not compact.

It is easy to see that the Lyapunov functions separate points, and that if $L$ is a Lyapunov function, we have $L(1 / n,-1 / n) \leq L(-1, n) \leq L(1 / n / 1 / n)$ so $L(-1, n) \rightarrow L(0,0)$ as $n \rightarrow \infty$. Thus the Lyapunov topology is weaker than the given topology on $X$.

If $(\hat{X}, \hat{f})$ is a Lyapunov compactification, then $\hat{X} \backslash X$ is a point $\hat{x}$ which is the limit of the sequence $\{(-1, n)\}$, so case (iv) of Theorem 7 occurs. The $\mathcal{G} \hat{f} \cap \mathcal{G} \hat{f}^{-1}$-equivalence class of $(0,0)$ is $\{(0,0), \hat{x}\}$.

Real actions. Theorem 14 applies to a real action $\left\{\varphi^{t}\right\}$. Let $I=[0,1]$, and write $\varphi$ for the closed relation $\varphi^{I}$, so $\varphi=\left\{(x, y) \in X \times X \mid y=\varphi^{t}(x)\right.$ for some $t$ with $0 \leq t \leq 1\}$.

Note that $\varphi$ is proper, and the Lyapunov functions for $\varphi$ are those continuous real-valued functions $L$ for which $L\left(\varphi^{t}(x)\right) \geq L(x)$ for $x \in X$ and $t \geq 0$, so these are exactly the class of functions as defined at the beginning of the paper. Therefore, if $\mathcal{G} \varphi \cap \mathcal{G} \varphi^{-1} \subset 1_{X}$, equivalently if the Lyapunov functions separate the points of the phase space $X$, then the Lyapunov topology coincides with the given topology.

Next, we want to consider those orbits on which all Lyapunov functions are constant, and relate these to generalized recurrence. This will require some modification of the definition, since $1_{X} \subset \varphi$ so every point is a generalized recurrent point for the relation $\varphi$.

Of course this does not really capture the idea of recurrence - namely of a point "returning to itself" in the long term. In order to achieve this, we consider two relations $\varphi^{J}$ (where $J=[1,2]$ ) and $\Lambda$, the prolongational limit relation. As we will see below, $\mathcal{G} \varphi^{J}$ and $\mathcal{G} \Lambda$ do not differ much from each other, but it is convenient to have both at hand.

Recall the definition of the prolongational limit relation $\Lambda:(x, y) \in \Lambda$ if there are sequences $\left\{x_{n}\right\}$ in $X$ and $\left\{t_{n}\right\}$ in $\mathbb{R}$ with $x_{n} \rightarrow x, t_{n} \rightarrow \infty$ and $\varphi^{t_{n}}\left(x_{n}\right) \rightarrow y$. The prolongational limit set $\Lambda(x)$ contains the omega limit set of $x$. Note that $|\Lambda|$ coincides with the non-wandering set of the flow.

It is immediate that the relation $\Lambda$ depends only on the orbit. That is, $\Lambda(x)=\Lambda\left(\varphi^{t}(x)\right)=\varphi^{t}(\Lambda(x))$ for all $t \in \mathbb{R}$ and the next lemma shows that the same holds for $\mathcal{G} \Lambda$. 
Lemma 15. If $x \in X$ and $t \in \mathbb{R}$, then $\varphi^{t} \mathcal{G} \Lambda(x)=\mathcal{G} \Lambda\left(\varphi^{t}(x)\right)=\mathcal{G} \Lambda(x)$.

Proof. For a fixed $t \in \mathbb{R}$, let the relation $\Sigma$ be defined by $(x, y) \in \Sigma$ if $y \in \varphi^{-t} \mathcal{G} \Lambda\left(\varphi^{t}(x)\right)$. Then $\Sigma$ is closed and transitive, and since $\Lambda \subset \Sigma$ we have $\mathcal{G} \Lambda \subset \Sigma$. It follows immediately that $\varphi^{t} \mathcal{G} \Lambda(x) \subset \mathcal{G} \Lambda\left(\varphi^{t}(x)\right)$ and equality follows by symmetry.

The equality with $\mathcal{G} \Lambda(x)$ can be proved by transfinite induction, but here is another proof. Note that the first part of this proof says that if $y \in \mathcal{G} \Lambda(x)$ then $\varphi^{s}(y) \in \mathcal{G} \Lambda\left(\varphi^{s}(x)\right)$ for all $s \in \mathbb{R}$.

Now let $K=\left\{(x, y) \mid\left(x, \varphi^{t}(y)\right) \in \mathcal{G} \Lambda\right.$ for all $\left.t \in \mathbb{R}\right\}$. Then $\Lambda \subset K$ and $K$ is closed and transitive. (For, if $(x, y),(y, z) \in K$ and $t \in R$, then $\left(x, \varphi^{t / 2}(y)\right.$ ) and $\left(y, \varphi^{t / 2}(z)\right)$ are in $\mathcal{G} \Lambda$ so $\left(\varphi^{t / 2}(y), \varphi^{t}(z)\right) \in \mathcal{G} \Lambda$ so $\left(x, \varphi^{t}(z)\right) \in \mathcal{G} \Lambda$.) Therefore $\mathcal{G} \Lambda \subset K$. That is, if $y \in \mathcal{G} \Lambda(x)$ and $t \in \mathbb{R}$, then $\varphi^{t}(y) \in \mathcal{G} \Lambda(x)$. Equivalently $\varphi^{t}(\mathcal{G} \Lambda(x)) \subset \mathcal{G} \Lambda(x)$, and applying $\varphi^{-t}$ equality follows.

LEMMA 16.

(i) $\mathcal{G}\left(\varphi^{J}\right)=\varphi^{[1, \infty)} \cup \mathcal{G}(\Lambda)$.

(ii) $\mathcal{G}(\varphi)=\varphi^{I} \cup \mathcal{G}\left(\varphi^{J}\right)$.

(iii) $\mathcal{G}(\varphi)=\varphi^{[0, \infty)} \cup \mathcal{G}(\Lambda)$.

(iv) $\mathcal{G} \varphi^{J}=\mathcal{G} \varphi^{J} \circ \varphi^{J} \cup \varphi^{J}=\mathcal{G} \varphi^{J} \circ \varphi^{I}=\varphi^{I} \circ \mathcal{G} \varphi^{J}$.

(v) If $t \in \mathbb{R}$ and $x \in X$, then $\varphi^{t} \mathcal{G} \varphi^{J}(x)=\mathcal{G} \varphi^{J} \varphi^{t}(x)$.

Proof. (i) Clearly $\Lambda \subset \mathcal{G}\left(\varphi^{J}\right)$, and it follows that the right side of (i) is contained in $\mathcal{G}\left(\varphi^{J}\right)$. Moreover the right side is closed and contains $\varphi^{J}$. It remains to show that it is transitive. This follows immediately from the previous lemma.

The proof of (ii) is similar (using (i)), and (iii) follows from (i) and (ii).

(iv) The second relation contains $\varphi^{J}$, is contained in $\mathcal{G} \varphi^{J}$, and is transitive and closed. The third relation contains $\mathcal{G} \varphi^{J}$ (since $\varphi^{I}$ is reflexive) and substituting from the second relation it equals $\left(\mathcal{G} \varphi^{J} \circ \varphi^{J} \cup \varphi^{J}\right) \circ \varphi^{I}$ which is contained in $\mathcal{G} \varphi^{J}$. The proof that the fourth relation equals $\mathcal{G} \varphi^{J}$ is similar. (Note that $\varphi^{I}$ and $\varphi^{J}$ are proper, so the various compositions are closed.)

(v) follows from (i) and Lemma 15.

TheOrem 17. Let $x \in X$. The following are equivalent:

(i) $x \in\left|\mathcal{G} \varphi^{J}\right|$.

(ii) $x \in|\mathcal{G} \Lambda|$.

(iii) $x$ is either a stationary point $\left(\varphi^{t}(x)=x\right.$ for all $\left.t \in \mathbb{R}\right)$ or there is $a y \neq x$ such that $x$ and $y$ are in the same $\mathcal{G} \varphi \cap \mathcal{G} \varphi^{-1}$-equivalence class as $x$.

(iv) There is a $t \neq 0$ such that $x$ and $\varphi^{t}(x)$ are in the same $\mathcal{G} \varphi \cap \mathcal{G} \varphi^{-1}$ equivalence class.

(v) Every Lyapunov function $\varphi$ is constant on the orbit of $x$. 
Proof. If (v) holds, then for all $t \in \mathbb{R}, x$ and $\varphi^{t}(x)$ are in the same $\mathcal{G} \varphi \cap \mathcal{G} \varphi^{-1}$-equivalence class, and it follows easily that (v) implies (i)-(iv).

Suppose (iv) holds. If $t>0$, and $L$ is a Lyapunov function, then $L\left(\varphi^{s}(x)\right)$ $=L(x)$ for all $s$ with $0 \leq s \leq t$ and (since $L \circ \varphi^{\tau}$ is a Lyapunov function for every real $\tau$ ) it follows immediately that $L$ is constant on the orbit of $x$. A similar argument works if $t<0$, so (iv) implies (v).

(Thus in fact all $\varphi^{t}(x)$ are $\mathcal{G} \varphi \cap \mathcal{G} \varphi^{-1}$-equivalent.)

Suppose (iii) holds. If $x$ is a stationary point, clearly the other four conditions hold. Otherwise there is a $y \neq x$ as stated in (iii). Again if $y$ is in the orbit of $x$ then (iv) holds. If not, by (iii) of Lemma 16, $y \in \mathcal{G} \Lambda(x)$ and $x \in \mathcal{G} \Lambda(y)$, so $x \in \mathcal{G} \Lambda(x)$. Therefore (ii) holds.

Recall that $\mathcal{G} \Lambda(x)$ is flow invariant, and $\mathcal{G} \Lambda\left(\varphi^{t}(x)\right)=\mathcal{G} \Lambda(x)$ for all $t \in \mathbb{R}$, so by Lemma 15, (ii) implies (iv).

If $x \in\left|\mathcal{G}\left(\varphi^{J}\right)\right|$, then (Lemma 16) either $x \in \varphi^{[1, \infty)}(x)$ in which case (iv) holds, or $x \in|\mathcal{G}(\Lambda)|$ and (ii) holds. Thus (i) implies the other conditions.

Condition (v) is analogous to the situation for continuous maps (Theorem 5).

A point satisfying the equivalent conditions in Theorem 17 is said to be a generalized recurrent point for the real flow $\left\{\varphi^{t}\right\}$.

COROllary 18. Consider the following conditions:

(i) $|\mathcal{G} \Lambda|=\emptyset$.

(ii) $\left|\mathcal{G} \varphi^{J}\right|=\emptyset$.

(iii) The Lyapunov functions for $\varphi$ separate points.

Then (i) and (ii) are equivalent and they imply (iii). If there are no stationary points then all three are equivalent.

Proof. The equivalence of (i) and (ii) follows from Theorem 17. If $x \neq y$ and $L(x)=L(y)$ for all Lyapunov functions $L$ then $x$ and $y$ are $\mathcal{G} \varphi \cap \mathcal{G} \varphi^{-1}$ equivalent, so by Theorem 17, $x \in|\mathcal{G} \Lambda|=\left|\mathcal{G} \varphi^{J}\right|$. Therefore (i) and (ii) imply (iii). If there are no stationary points and (iii) holds, then since the Lyapunov functions determine the $1_{X} \cup\left(\mathcal{G} \varphi \cap \mathcal{G} \varphi^{-1}\right)$-equivalence classes, it follows from (iii) of Theorem 17 that (i) and (ii) hold.

The proof of Theorem 2. The following, which is a special case of a theorem of Nachbin [4, is the main step.

TheOrem 19. Let $X_{0}$ be a compact subset of a locally compact $\sigma$-compact space $X$, and let $R$ be a closed transitive relation on $X$. Let $R_{0}=R \cap$ $\left(X_{0} \times X_{0}\right)$. If $L_{0}: X_{0} \rightarrow[a, b]$ is a Lyapunov function for $R_{0}$ then there exists a Lyapunov function $L: X \rightarrow[a, b]$ for $R$ which extends $L_{0}$. 
Proof. In the proof, we will write $A \subset \subset B$ to mean that the closure of $A$ is contained in the interior of $B$.

It is sufficient to prove Theorem 19 under the assumption that $X$ is compact. For, once this is accomplished, let $\left\{K_{n}\right\}$ be a sequence of compact subsets of the locally compact $\sigma$-compact space $X$ whose union is $X$ with $X_{0}=K_{0}$ and $K_{n-1} \subset \subset K_{n}$. Let $R_{n}=R \cap\left(K_{n} \times K_{n}\right)$, and apply the result for compact spaces inductively to extend the $R_{n}$-Lyapunov function $L_{n}$ to an $R_{n+1}$-Lyapunov function $L_{n+1}$ on $K_{n+1}$. The union of the functions $L_{n}$ is the required extension $L$. Note that $L$ is continuous on $L$ because each $K_{n+1}$ is a neighborhood of $K_{n}$.

Now suppose that $X$ is compact.

We can assume $a=0$ and $b=1$. By replacing $R$ by $R \cup 1_{X}$ and $R_{0}$ by $R_{0} \cup 1_{X_{0}}$ we can assume that $R$ and $R_{0}$ are reflexive as well as transitive.

We mimic the proof of Urysohn's Lemma. Let $\Lambda$ be an enumeration of the rational numbers in $[0,1]$ with $\lambda_{0}=0, \lambda_{1}=1$.

We will define, for $\lambda \in \Lambda$, sets $B_{\lambda}$ satisfying

$$
\begin{gathered}
R\left(B_{\lambda}\right)=B_{\lambda}, \quad R\left(L_{0}^{-1}((\lambda, 1])\right) \subset \subset\left(B_{\lambda}\right), \quad R\left(L_{0}^{-1}([\lambda, 1])\right) \subset B_{\lambda} \\
R^{-1}\left(L_{0}^{-1}([0, \lambda))\right) \cap B_{\lambda}=\emptyset \quad \text { and } \quad \lambda^{\prime}>\lambda \text { implies } B_{\lambda^{\prime}} \subset \subset B_{\lambda} .
\end{gathered}
$$

Let $B_{1}=R\left(L_{0}^{-1}(1)\right)$ and $B_{0}=X$. Notice that for all $\lambda$,

$$
R\left(L_{0}^{-1}([\lambda, 1])\right) \cap R^{-1}\left(L_{0}^{-1}([0, \lambda))\right)=\emptyset,
$$

since $R$ is transitive with restriction $R_{0}$ and $L_{0}$ is an $R_{0}$-Lyapunov function.

In the induction which follows, we will make use of the following fact, which is a direct consequence of compactness. If $C$ is a closed set with $R(C)=C$ and $U$ is an open set containing $C$, then there is a closed set $C_{1}$ with $C_{1} \subset U, R\left(C_{1}\right)=C_{1}$, and $C$ contained in the interior of $C_{1}$.

Assume that $B_{\lambda}$ has been defined for all $\lambda$ in $\Lambda_{n}=\left\{\lambda_{i}: i=0, \ldots, n\right\}$ with $n \geq 1$. Let $\lambda=\lambda_{n+1}$ and let $\lambda^{\prime}<\lambda<\lambda^{\prime \prime}$ be the nearest points in $\Lambda_{n}$ below and above $\lambda$.

Choose a sequence $\left\{t_{n}^{-}\right\}$with $t_{0}^{-}=\lambda^{\prime}$, increasing with limit $\lambda$, and $\left\{t_{n}^{+}\right\}$ with $t_{0}^{+}=\lambda^{\prime \prime}$, decreasing with limit $\lambda$.

Define $Q_{0}^{-}=B_{\lambda^{\prime}}$ and $Q_{0}^{+}=B_{\lambda^{\prime \prime}}$. Inductively, choose $Q_{n}^{+}$and then $Q_{n}^{-}$ for $n=1,2, \ldots$ so that $R\left(Q_{n}^{ \pm}\right)=Q_{n}^{ \pm}$and

$$
\begin{gathered}
R\left(L_{0}^{-1}\left(\left[t_{n}^{+}, 1\right]\right)\right) \cup Q_{n-1}^{+} \subset \subset Q_{n}^{+} \subset \subset Q_{n-1}^{-} \backslash R^{-1}\left(L_{0}^{-1}([0, \lambda])\right), \\
R\left(L_{0}^{-1}([\lambda, 1])\right) \cup Q_{n}^{+} \subset \subset Q_{n}^{-} \subset \subset Q_{n-1}^{-} \backslash R^{-1}\left(L_{0}^{-1}\left(\left[0, t_{n}^{-}\right]\right)\right) .
\end{gathered}
$$

Finally, define

$$
B_{\lambda}=\bigcap_{n} Q_{n}^{-} \quad \text { so that } \quad B_{\lambda} \supset \bigcup_{n} Q_{n}^{+} .
$$

It is easy to check that $B_{\lambda}$ satisfies the required conditions, thus extending the definitions to $\Lambda_{n+1}$. By induction they can be defined on all of $\Lambda$. 
Having defined the $B_{\lambda}$ 's we proceed as in Urysohn's Lemma to define $L(x)$ by the Dedekind cut associated with $x$. That is,

$$
L(x)=\inf \left\{\lambda: x \notin B_{\lambda}\right\}=\sup \left\{\lambda: x \in B_{\lambda}\right\} .
$$

Continuity follows as in Urysohn's Lemma. Because each $B_{\lambda}$ is $R$-invariant, $L$ is a Lyapunov function. The additional conditions on these sets imply that if $x \in X_{0}$ then $x \in B_{\lambda}$ if and only if $\lambda \leq L_{0}(x)$. Hence, $L$ is an extension of $L_{0}$.

Now we can prove Theorem 2. Let $\left\{K_{n}\right\}$ be a sequence of compact sets as in the proof of Theorem 19 with $K_{0}=\emptyset$ and let $R_{n}$ be as in that proof. For $n=0,1, \ldots$ let

$$
K_{n+1 / 2}=K_{n} \cup\left[(A \cup B) \cap K_{n+1}\right], \quad R_{n+1 / 2}=R \cap\left(K_{n+1 / 2} \times K_{n+1 / 2}\right) .
$$

For $K_{1 / 2}=(A \cup B) \cap K_{1}$, let $L_{1 / 2}$ be 1 on $A$ and 0 on $B$, so $L_{1 / 2}$ is obviously a Lyapunov function on $K_{1 / 2}$. Apply Theorem 19 to obtain a Lyapunov function $L_{1}$ on $K_{1}$. Inductively, if $L_{n}$ is an $R_{n}$-Lyapunov function on $K_{n}$ which is 1 on $A \cap K_{n}$ and 0 on $B \cap K_{n}$ obtain an $R_{n+1 / 2}$-Lyapunov function $L_{n+1 / 2}$ on $K_{n+1 / 2}$ by defining it to be 1 and 0 on the new points of $A$ and $B$ respectively. Now apply Theorem 19 to obtain an $R_{n+1}$-Lyapunov function $L_{n+1}$ on $K_{n+1}$. Finally $L$ is the union of the functions $L_{n}$.

\section{References}

[1] E. Akin, The General Topology of Dynamical Systems, Amer. Math. Soc., Providence, 1993.

[2] E. Akin and J. Auslander, Compactifications of dynamical systems, arXiv:1004.0323 (2010).

[3] J. Auslander, Generalized recurrence in dynamical systems, Contr. Differential Equations 3 (1964), 69-74.

[4] L. Nachbin, Topology and Order, Van Nostrand, Princeton, 1965.

Ethan Akin

Department of Mathematics

City College of New York

New York, NY 10031-9100, U.S.A.

E-mail: ethanakin@earthlink.net
Joseph Auslander Department of Mathematics University of Maryland College Park, MD 20742-0001, U.S.A. E-mail: jna@math.umd.edu

Received May 11, 2010

Revised version August 12, 2010 